\title{
Low-frequency noise behavior at reverse bias region in InAs/GaSb superlattice photodiodes on mid- wave infrared
}

T. Tansel

K. Kutluer

A. Muti

Ö. Salihoglu

A. Aydinli

R. Turan 


\title{
Low-frequency noise behavior at Reverse Bias Region in InAs/GaSb Superlattice Photodiodes on Mid-Wave Infrared
}

\author{
T. Tansel ${ }^{\text {a }}$, K. Kutluer ${ }^{\text {a }}$, A. Muti ${ }^{\text {b }}$, Ö. Salihoglu ${ }^{\text {b }}$, A. Aydinli ${ }^{\text {b }}$, and R. Turan ${ }^{\text {a }}$ \\ ${ }^{a}$ Middle East Technical University (METU), Department of Physics, 06531 Ankara/Turkey \\ ${ }^{\mathrm{b}}$ Bilkent University, Department of Physics, Advanced Research Laboratory, 06800 Ankara/ Turkey
}

\begin{abstract}
We describe a relationship between the noise characterization and activation energy of InAs/GaSb superlattice MidWavelength-Infrared photodiodes for different passivation materials applied to the device. The noise measurements exhibited a frequency dependent plateau (i.e. 1/f-noise characteristic) for unpassivated as well as $\mathrm{Si}_{3} \mathrm{~N}_{4}$ passivated samples whereas 1/f-type low noise suppression (i.e. frequency independent plateau) with a noise current reduction of more than one order of magnitude was observed for $\mathrm{SiO}_{2}$ passivation. For reverse bias values below $-0.15 \mathrm{~V}$, the classical Schottky-noise calculation alone did not appear to describe the noise mechanism in a SL noise behavior, which shows a divergence between theoretically and experimentally determined noise values. We identify that, the additional noise appears, with and without passivation, at the surface activation energy of $<60 \mathrm{meV}$ and is inversely proportional to the reverse bias. This is believed to be caused by the surface dangling-bonds (as well as surface states) whose response is controlled by the applied reverse bias. The calculated noise characteristics showed a good agreement with the experimental data.
\end{abstract}

Keywords: Mid-Wave-Infrared Photodiode, Passivation, Noise Characterization, InAs/GaSb, surface activation energy

\section{INTRODUCTION}

In recent years, Mid-Wavelength-Infrared (MWIR) InAs/GaSb-superlattice (SL) photodetectors are attracting considerable attention due to its promises for new generation infrared detectors having applications in military, industrial, and medical technologies. When compared with the $\mathrm{HgCdTe}$ photodiodes which dominate current IR detector technology, MWIR SL photodetectors offer remarkable advantages such as low noise current due to the suppression of Auger recombination [1, 2, 21]. However, in spite of numerous studies on different features SL detectors, the effects of surface leakage current on the detector performance are not well understood. In general, the existence of dangling- bonds at the crystal surface -attributed to the abrupt termination of semiconductor surface- and native oxides on the SL leading to surface leakage current, and that can extremely limit/degrade the SL infrared detector performance. This might cause to form a favorable surface potential for easy carrier transport over the surface [2,3]. Hence, without proper surface passivation the leakage current is significant, particularly on the mesa side-walls. This is of high importance especially where the detector area approaches the sizes needed for focal- plane -array applications. Consequently, intentional passivation is needed to suppress the leakage current by minimizing/eliminating the states and hence the influence of foreign materials [2]. The relation between surface leakage and the noise properties of the detector needs to be clarified for a complete understanding. Certainly, noise experiments shall guide one to calculate the true specific detectivity as well. The noise figure performance, which is an indicator of the quality of a photodetector, is one of the most sensitively affected parameters by the surface and bulk conditions in semiconductor material [3,4]. Therefore, in this work, we used the SLs noise characteristics and performed direct measurements of the dark noise current vs. passivation material with a view to understand the effects of dark current and noise on the detector performance.

In most device applications, insulating materials such as silicon nitride $\left(\mathrm{Si}_{3} \mathrm{Ni}_{4}\right)$ or silicon dioxide $\left(\mathrm{SiO}_{2}\right)$ are commonly applied as a passivation layer, which ensures the device is electrically insulated from the ambient atmosphere [5]. Here we reveal the surface effects by studying the relationship between the spectral noise level and the passivation as well as without passivation material on a MWIR InAs/GaSb SL photodetector and describe the surface limited current mechanism under reverse bias conditions.

Infrared Technology and Applications XXXIX, edited by Bjørn F. Andresen, Gabor F. Fulop,

Charles M. Hanson, Paul R. Norton, Proc. of SPIE Vol. 8704, 87040Y · (c) 2013 SPIE

CCC code: $0277-786 \mathrm{X} / 13 / \$ 18 \cdot$ doi: $10.1117 / 12.2016388$ 


\section{EXPERIMENTAL}

In order to study the surface effects on the MWIR InAs/GaSb SL detector structures were obtained from IQE Inc. (with cut-off wavelength $4.9 \mu \mathrm{m}$ at $79 \mathrm{~K}$ ). The device structures were grown by molecular beam epitaxy on an n-type (001) GaSb substrate, based on 60-periods n-type/60-periods non- intentionally doped (n.i.d.) active region/90-periods p-type InAs/GaSb SL. Each period in the entire structure consists of $2.85 \mathrm{~nm}$ InAs and $3.3 \mathrm{~nm}$ GaSb layers with InSb-like interfaces. GaSb layers in the p-type superlattice period were doped with Be to a level of $1.5 \times 10^{17} \mathrm{~cm}^{-3}$ while the InAs layers were doped with Te to a level of $5 \times 10^{17} \mathrm{~cm}^{-3}$. The entire SL structure was enclosed by a $20 \mathrm{~nm}$ n-type InAs: Te $\left(5 \times 10^{17} \mathrm{~cm}^{-3}\right)$ top and a $1 \mu \mathrm{m}$ thick p-type GaSb: $\mathrm{Be}\left(1 \times 10^{17} \mathrm{~cm}^{-3}\right)$ bottom contact layers.

To protect the sample surface (with a mesa size of $400 \mu \mathrm{m} \times 400 \mu \mathrm{m}$ ) from ambient atmosphere, surface passivation was realized directly after the wet etching (which uses phosphoric and citric acid solutions) with a $230 \mathrm{~nm} \mathrm{SiO}_{2}$ or 150 $\mathrm{nm} \mathrm{Si}_{3} \mathrm{Ni}_{4}$ coating applied by Plasma Enhanced Chemical Vapor Deposition (PECVD) method [22].

A Ti-Au (30nm/300nm) bi-layer was evaporated onto the top and back surfaces of the photodiodes for ohmic contacts.

In order to measure the temperature dependent dark current or resistance- area product (RA), a CTI Cryogenics helium cryostat (22C Cryodyne Refrigeration System) was employed as cold head and a Keithley (Keithley 6487) picoammeter was used to apply the DC bias (Vbias) to the detector and to read the output current.

For the dark current noise characterizations, the specimen was placed in a cryostat and cooled down to $79 \mathrm{~K}$ using liquid nitrogen. During the measurements, all instruments including the low noise current amplifier (Stanford Research Systems, SR570), cryostat and FFT spectrum analyzer (Stanford Research system, SR760) were placed inside a shielded box. We subtracted the system noise from the measured total noise (in $\mathrm{A} / \sqrt{\mathrm{Hz}}$ ) to obtain the intrinsic detector noise6. Here, the intrinsic detector noise sources consist of white noise as well as $1 / f-$-noise [7]. Schottky-noise $\left(\mathrm{I}_{\mathrm{sn}}^{2}=2 \mathrm{qI}\right)$ and Johnson-noise $\left(\mathrm{I}^{2}{ }_{\mathrm{jn}}=4 \mathrm{k}_{\mathrm{B}} \mathrm{T} / \mathrm{R}_{\mathrm{d}}\right)$ together form the 'white noise' $\left(\mathrm{I}^{2}{ }_{\mathrm{sn}}+\mathrm{I}^{2}{ }_{\mathrm{jn}}\right)$ of the detector, where $\mathrm{k}_{\mathrm{B}}$ the Boltzmann constant, $\mathrm{T}$ the temperature, $\mathrm{R}_{\mathrm{d}}$ the dark differential resistance, $\mathrm{q}$ the electronic charge constant, and $\mathrm{I}$ the dark current. The $1 / \mathrm{f}$-noise is inversely proportional to frequency and so it is above the white noise level at low frequencies. In general, this type of $1 / \mathrm{f}$-noise is particularly sensitive to surface imperfections [3,4].

\section{RESULT AND DISCUSSION}

The dark current-voltage $(\mathrm{I}-\mathrm{V})$ characteristics at $\mathrm{T}_{\text {sample }}=79 \mathrm{~K}$ for $\mathrm{SL}$ diodes with variable passivation layers and unpassivated surface are shown in Figure 1. At Vbias $=-0.5 \mathrm{~V}$ the unpassivated and $\mathrm{Si}_{3} \mathrm{~N}_{4}$ passivated samples exhibits a maximum dark current density of, $\sim 10^{-5}$ and $\sim 10^{-4} \mathrm{~A} / \mathrm{cm}^{2}$ respectively, while the $\mathrm{SiO}_{2}$ passivated device shows a nearly constant line with a dark current density of $\sim 10^{-7} \mathrm{~A} / \mathrm{cm}^{2}$ at the overall reverse bias regime. The dark current density was seen to increase approximately proportional to $\mathrm{V}_{\text {bias }}$ for the unpassivated and $\mathrm{Si}_{3} \mathrm{Ni}_{4}$ passivated devices. However, in comparison, the best results were presented by the $\mathrm{SiO}_{2}$ passivated device with the dark current density reduced by up to 2 to 3 orders of magnitude. From this follows, the $\mathrm{SiO}_{2}$ passivation is likely to be the best candidate to minimize the surface leakage current [8]. 


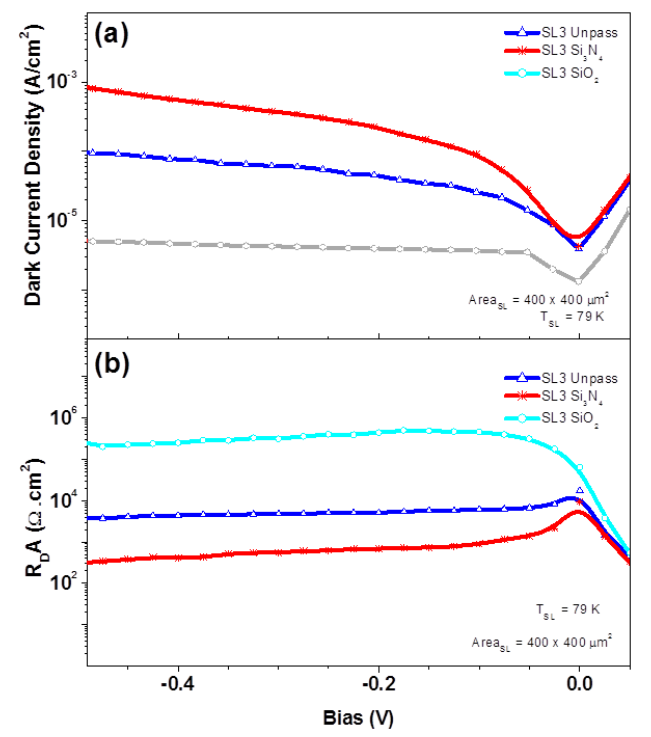

Figure 1. Dark current density vs. applied bias voltage at $\mathbf{T}_{\text {sample }}=79 \mathrm{~K}$ for $\mathrm{SL}$ devices with (a) no intentional passivation, passivated by $\mathrm{Si}_{2} \mathrm{~N}_{3}$, passivated by $\mathrm{SiO}_{3}$. At $-0.5 \mathrm{~V}$ bias voltage, the unpassivated, $\mathrm{Si}_{3} \mathrm{~N}_{4}$ and $\mathrm{SiO}_{2}$ passivated samples exhibit a dark current density of $1 \times 10^{-4} \mathrm{~A} / \mathrm{cm}^{2}, 8 \times 10^{-4} \mathrm{~A} / \mathrm{cm}^{2}$, and $4 \times 10^{-6} \mathrm{~A} / \mathrm{cm}^{2}$, respectively. RA vs. Bias results in part (b), the effect of passivation layers can be also observed in $R A$ values at $T_{\text {sample }}=79 \mathrm{~K}$ for unpassivated, $\mathrm{SiO}_{2}$, and $\mathrm{Si}_{3} \mathrm{~N}_{4}$ passivated samples respectively $\left(17 \mathrm{k} \Omega . \mathrm{cm}^{2}, 64 \mathrm{k} \Omega . \mathrm{cm}^{2}\right.$ and $\left.9 \mathrm{k} \Omega . \mathrm{cm}^{2}\right)$. As in the dark current density results, there are not so much differences in $R_{0} A$ values. However differences between values increase drastically with creasing reverse bias.

In order to appreciate the above offset in the I-V as well as RA-V characteristics (refer to Figure 1), we studied the dark noise characteristic of the SL sample over the 1 to $100 \mathrm{~Hz}$ frequency range at

$\mathrm{V}_{\text {bias }}=-0.1 \mathrm{~V}$ and $\mathrm{T}_{\text {sample }}=79 \mathrm{~K}$.

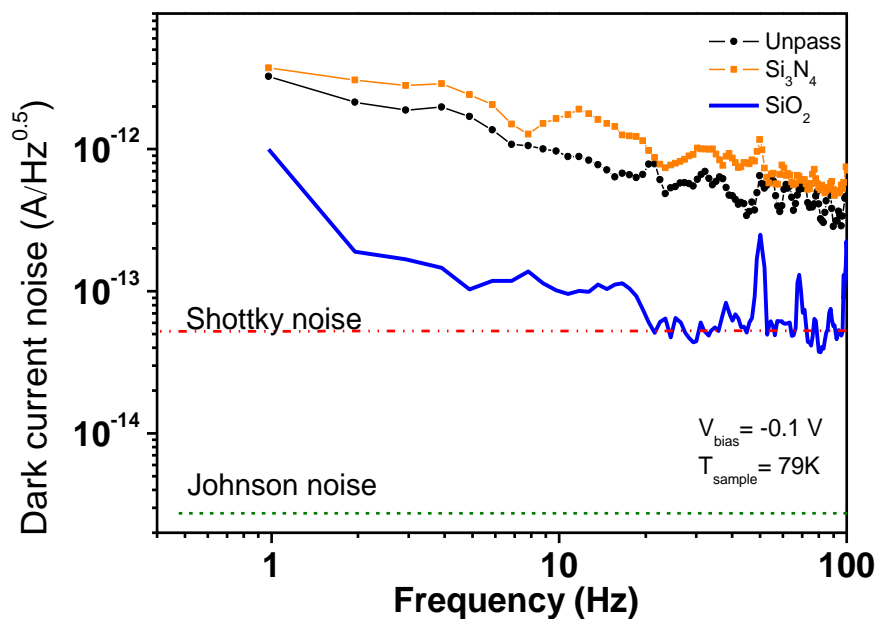

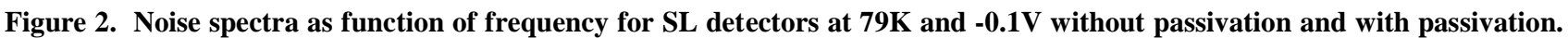
The peaks at $50-100 \mathrm{~Hz}$ are due to electronic noise and its harmonics [6]. 
Figure 2 shows the noise spectra for unpassivated, and $\mathrm{SiO}_{2}$ passivated SLs. For $\mathrm{SiO}_{2}$ passivated sample, the frequency independent plateau in the $20-100 \mathrm{~Hz}$ range is due to white-noise. This is well matched with the calculated theoretical Schottky-noise $\left(\mathrm{I}_{\mathrm{sn}}=5 \times 10^{-14} \mathrm{~A} / \sqrt{\mathrm{Hz}}\right)$, and thus indicates a good passivation level.

The 1/f-noise is suppressed and the dark noise current is reduced by one order of magnitude (at $\mathrm{V}_{\text {bias }}=-0.1 \mathrm{~V}$ ) against to unpassivated SL. The effect of surface states and foreign materials seem to be suppressed along the exposed mesa Surface through $\mathrm{SiO}_{2}$ passivation. In contrast, the noise values for other materials (unpassivated as well as $\mathrm{Si}_{3} \mathrm{~N}_{4}$ passivated SL) reveal frequency dependent behaviors in the same frequency range, which indicate a $1 / \mathrm{f}$-noise contribution. The existence of the 1/f-noise contribution approves that $\mathrm{Si}_{3} \mathrm{~N}_{4}$ passivation did not lead to an enhancement but rather degradation of the sample quality. The dark noise current in an ordinary diode often increases under reverse bias due to 1/f-noise in the surface leakage current [9].

The noise spectra of $\mathrm{SiO}_{2}$ passivated detector displayed in Figure 3 do not exhibit additional noise at $-0.1 \mathrm{~V}$ at $\sim 30 \mathrm{~Hz}$ (i.e. the dominant noise is Schottky-noise), but at $-0.5 \mathrm{~V}$ an additional noise seems to increase with an applied reverse $\mathrm{V}_{\text {bias. }}$ This appears to have resulted from an additional frequency dependent noise (potentially $1 / \mathrm{f}-$ noise). From the observed interdependence, such 1/f-noise should be related to the surface leakage current. The disagreement (seen in Figure 3) with the theoretical Schottky noise expectation (by a factor of $\sim 3$ ) at $-0.5 \mathrm{~V}$ is possibly caused by the $1 / \mathrm{f}$-noise which further increases with the reverse bias-which in fact is in agreement to that reported in Ref. 6 and 7.

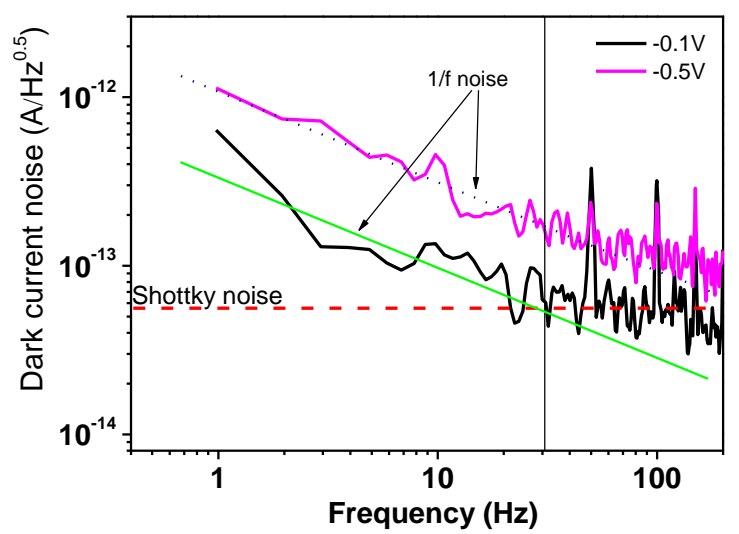

Figure 3. Noise spectra as function of frequency for $\mathrm{SiO} 2$ passivated devices at $\mathbf{- 0 . 1 V}$ and $\mathbf{- 0 . 5 \mathrm { V }}$ (temperature $\mathbf{7 9 K}$ ). The fitted strait line and dotted lines indicates the $1 / \mathrm{f}$-noise behavior.

In order to verify the effect of surface leakage current, we performed dark I-V measurements on a series of diodes with different perimeter-to-area ratio, since the surface resistivity is directly proportional to the surface-dependent leakage current of the diodes for unpassivated SL [8]. However, the measured surface resistivity of $\sim 1 \times 10^{6} \Omega \mathrm{cm}$ for $\mathrm{SiO}_{2}$ passivated SL diode at the unbiased condition is relatively too large to conclude to a self-initiated surface leakage current.

Another method to obtain the dominating mechanism at the considered temperature range is to measure the RA vs. reciprocal temperature $(1000 / \mathrm{T})$ which can separate the bulk current from surface current dynamic of the diodes [10].

In order to do this, first of all it should be verified that at temperature range around $80 \mathrm{~K}$, the dominant current mechanism is surface limited current. Temperature dependent I-V measurements and Arrhenius Plot analysis provides evidence for dominated current mechanisms. In Figure 4, results of temperature dependent I-V measurements in the form of resistance vs. bias are given for temperature range from 11 to $250 \mathrm{~K}$ for $\mathrm{SiO}_{2}$ passivated SL sample. The effect of temperature on resistance of the photodiode is clearly seen. 

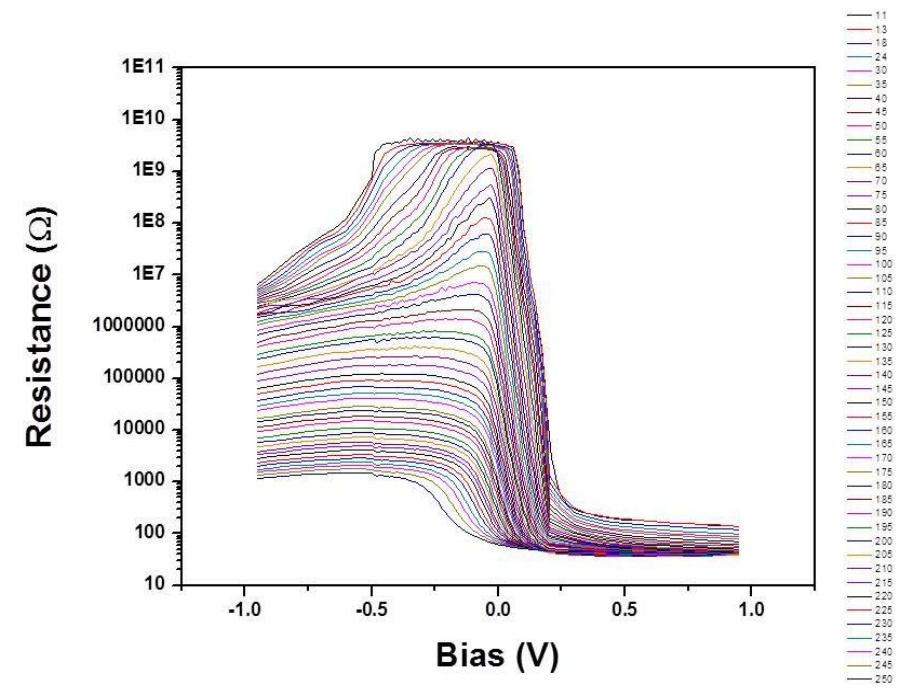

Figure 4. Resistance vs. Bias results from $11 \mathrm{~K}$ to $250 \mathrm{~K}$ temprature range for $\mathrm{SiO}_{2}$ passivated sample.

This measurement is also realized for unpassivated and $\mathrm{Si}_{3} \mathrm{~N}_{4}$ passivated SL samples. For each temperature, resistance values at specific bias values are extracted from the data to plot resistance as a function of inverse temperature graph as presented in Figure 5. In this graph, 3 different current mechanisms are plotted for different temperature regions.

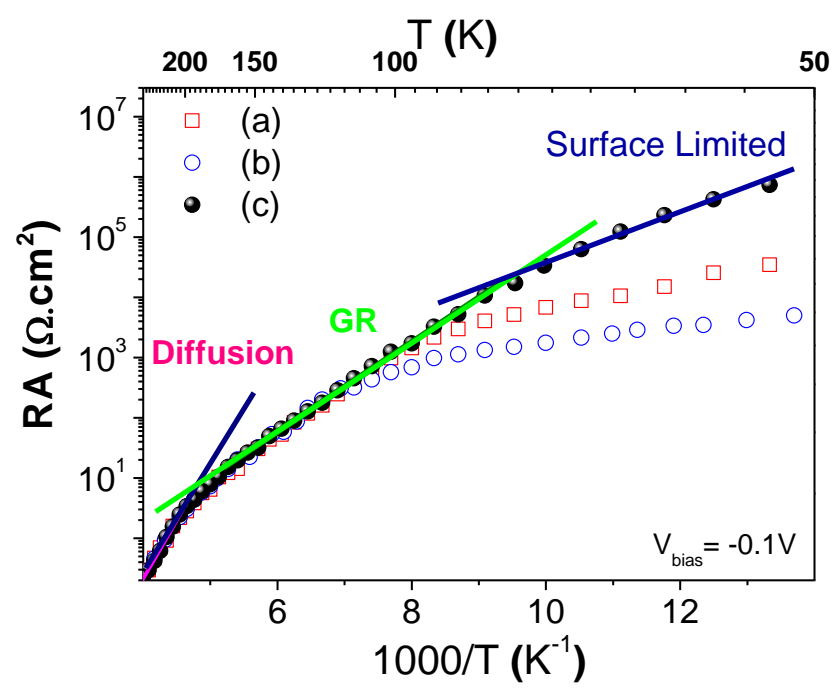

Figure 5. Resistance-area product vs. Temperature dependent for the $\mathrm{SL}$ device passivated with $\mathrm{SiO}_{2}$ at the different Bias voltages of - 0.1 and $-0.5 \mathrm{~V}$.

The RA vs. 1000/T results (see Figure 5) shows three distinct regimes with different dominant current mechanisms: diffusion from $150-200 \mathrm{~K}$, generation-recombination (GR) from $95-150 \mathrm{~K}$, and surface limited current at < $95 \mathrm{~K}$; at the low temperatures the deviation from the Arrhenius fit indicates the surface leakage current $[9,-13]$. 
At temperature values higher than $200 \mathrm{~K}$, thermal energies of charge carriers are sufficient to realize band-to-band transition. Therefore the first slop of Arrhenius plot indicates the diffusion current mechanism.

From the slope of Figure 5, activation energy $\left(\mathrm{E}_{\text {act }}\right)$ required in band-to-band transition can be calculated which is also equal to the band gap of the device obtained from optical measurements. It is found $0.24 \mathrm{eV}$, very close to optical measurements. The GR $\mathrm{E}_{\text {act }}$ is $0.12 \mathrm{eV}$ (e.g. for $\mathrm{SiO}_{2}$-passivated sample), which is half of the band gap as expected in the literature [15].

For different passivation layers, a different deviation from Arrhenius fit was observed for 65 - $95 \mathrm{~K}$. In this temperature range, the dominating current mechanism is the surface limited current [10], which is somewhat suppressed in the $\mathrm{SiO}_{2}$ sample in contrast to other samples at zero bias. In other words, the deviation which may corresponds to surface leakage current from the Arrhenius fit to the I-V curves for different reverse biases in the temperature range of 65 - $95 \mathrm{~K}$ (see Figure 6).

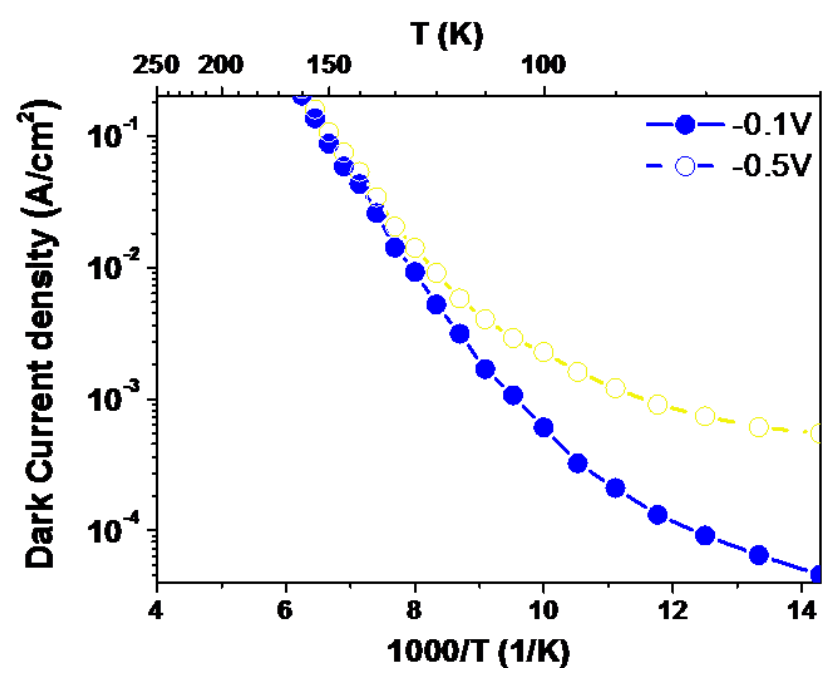

Figure 6. Dark current density as function of inverse Temperature for passivated by $\mathrm{Si}_{3} \mathrm{~N}_{4} \mathrm{samples}$ at the different bias

In regards to Ref.10, the higher the reverse bias the more the surface states will form, since surface leakage current is a direct result of states, and consequently the surface states are proportional to the applied reverse $\mathrm{V}_{\text {bias. }}$. Therefore, by considering the results of Figure 3 and Figure 5, we can relate the surface states to the 1/f-noise as well as additional noise in Figure 8.

In order to better understand the influence of bias voltage on surface states, we investigate that in terms of the activation energy. The activation energy of the current limiting factors at different reverse bias values can be determined from the slope of the linear fit to $\ln (\mathrm{RA})$ vs. 1000/T for different reverse voltages (see Figure 7) [14,15].

Figure 6 shows the $\mathrm{E}_{\text {act }}$ vs. reverse $\mathrm{V}_{\text {bias }}$ for all SL diodes. Clearly, an inverse dependence is seen between $\mathrm{E}_{\text {act }}$ and reverse $\mathrm{V}_{\text {bias. }}$. Lowering $\mathrm{E}_{\text {act }}$ implies that the increment of the surface states is caused by the reverse bias voltage and this can be attributed to the appearance of additional noise or 1/f-noise in the experimental results. This observation is also valid for the sample with no passivation. 


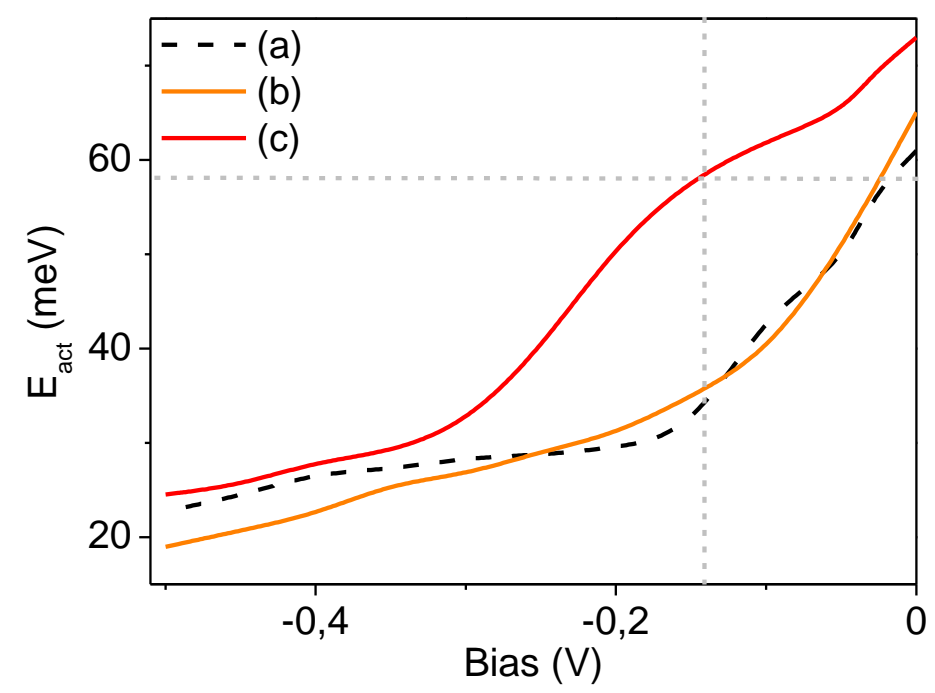

Figure 7. Activation energy vs. applied reverse bias for (a) unpassivated, (b) $\mathrm{Si}_{3} \mathrm{~N}_{4}$ passivated and (c) $\mathrm{SiO}_{2}$ passivated devices. The activation energy decreases with applied reverse bias [22].

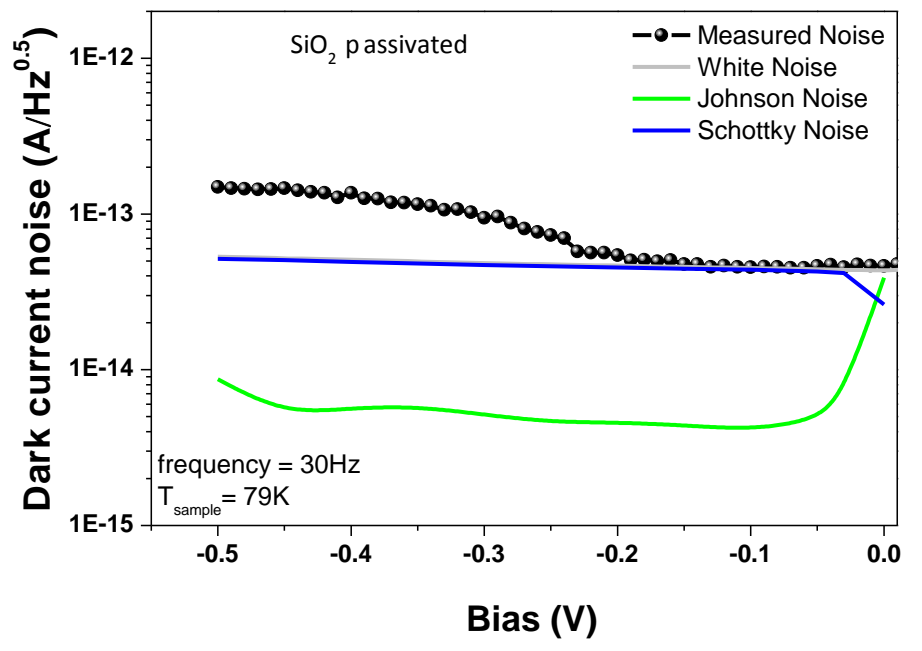

Figure 8. Dark current noise with respect to bias voltage for $\mathrm{SiO}_{2}$ passivated $\mathrm{SL} 3$ sample

In the following, for sake of clarity/brevity, we discuss the experimental results obtained on $\mathrm{SiO}_{2}$ passivated samples. A cross correlation between Figure 7 and Figure 8 indicates that for $\mathrm{E}_{\text {act }}$ higher than $60 \mathrm{meV}$ the Schottky-noise dominates at the smaller reverse $\mathrm{V}_{\text {bias }}$ than $-0.1 \mathrm{~V}$. However, for $\mathrm{E}_{\text {act }}$ approximately smaller than $60 \mathrm{meV}$, the dominating type is $1 / \mathrm{f}$-noise at the higher reverse $\mathrm{V}_{\text {bias }}$ than $-0.15 \mathrm{~V}$. Further, it results in much higher noise in the detector (i.e. at $\sim 30 \mathrm{~Hz}$ frequency) as seen in Figure 3 as well as in Figure 8.

More specifically, the value of surface potential-(energy barrier) can be adjusted via bias voltage (by an amount of electrostatic energy) which alters the surface charge equilibrium between the surface states and space charges. With increasing reverse bias, a decrease in the activation energy barrier is expected and so more states, as well as surface states [16], would be generated at the semiconductor interface or insulator interface and more electrons should recombine. More accurately, these should occur at the critical barrier height of $<60 \mathrm{meV}$ seen in Figure 7. In short, increment of states with applied reverse bias causes more surface recombination current which acts as an independent noise source. This $1 / \mathrm{f}$-noise source is dominant at the activation energies corresponding to the bias voltages lower than $60 \mathrm{meV}$. Due to the fact that this noise source is the consequence of recombination process of charge carriers on the 
surface, no additional current flow can be seen in I-V characteristic. This means that the charge carriers do not leave the device, although they flow to the surface, they are lost on the surface and create noise. But recombination noise is directly related with the carrier concentration on the surface.

The value of carrier concentration $\left(\mathrm{n}_{\mathrm{s}}\right)$ is strongly dependent on the operation bias voltages at constant temperature, and leads to the exponential behavior of $E_{\text {act, }}$ which results in the analytical expression $n_{s} \sim \exp \left(-E_{a c t} / k_{B} T\right)$ where $n_{s}$ is the charge carrier concentrations $[15,16]$. By assuming, $\mathrm{n}_{\mathrm{s}}$ are proportional to the leakage current $\mathrm{I}_{\mathrm{lc}}$ with a proportionality factor of $I_{c}$. The final equation is formed as $I_{l c}=I_{c} \exp \left(-E_{a c t} / k_{B} T\right)$.

Under the assumption that above process behaves as a photovoltaic device mechanism, hence to estimate the surface leakage current noise $\mathrm{I}_{\mathrm{lcn}}$ similar like Schottky-noise calculation for $\mathrm{p}$-n junction diodes [17, 19], is given by

$$
I_{l c n}^{2}=2 q I_{c} \exp \left(-\frac{E_{a c t}}{k_{B} T}\right)
$$

By claiming that all the noise sources (i.e. $\mathrm{I}_{\mathrm{JN}}, \mathrm{I}_{\mathrm{SN}}$ and $\mathrm{I}_{\mathrm{lcn}}$ ) are statistically independent, the total noise $\mathrm{I}_{\text {total }}$ is then found as

$$
\mathrm{I}_{\text {total }}^{2}=\mathrm{I}^{2}{ }_{\mathrm{SN}}+\mathrm{I}_{\mathrm{JN}}^{2}+\mathrm{I}_{\text {len }}^{2}
$$

To demonstrate the validity of equation (3), fits of experimental noise dark current were made in the voltage range $0-0.5$ $\mathrm{V}$ to identify the bias-dependent dominant noise dark current component.

The parameters were deduced from experimental data such as $E_{\text {act }}, \mathrm{I}_{\mathrm{SN}}, \mathrm{I}_{\mathrm{JN}}$ and $\mathrm{I}_{\mathrm{c}}$. Wherein, the $\mathrm{I}_{\mathrm{c}}$ was deduced from $\mathrm{I}_{\text {len }}^{2}$ (i.e., is formed as $\mathrm{I}_{\text {lcn }}^{2}=\mathrm{I}_{\text {total- }}^{2} \mathrm{I}_{\mathrm{SN}}^{2}-\mathrm{I}_{\mathrm{JN}}^{2}$ ) vs. $\mathrm{E}_{\text {act }}$ plot graphically by curve fitting, i.e., curve fitting was used to determine the value of $I_{c}$ which is the intercept (where $E_{\text {act }}$ is zero) divided by $2 q$ at around $79 \mathrm{~K}$ [20]. More precisely, $I_{c}$ is the total number of surface charge carrier concentration (which may lead to a recombination) per unit time and $\exp \left(-\mathrm{E}_{\mathrm{act}} / \mathrm{k}_{\mathrm{B}} \mathrm{T}\right)$ is the probability that any given surface carrier concentration will result in a recombination in the surface states. The temporal fluctuation in recombination rate per unit time itself leads to frequency dependence behavior which in turn results in surface recombination noise $\mathrm{I}_{\mathrm{lcn}}$. We propose inverse frequency dependence-but it is not clear exactly how the frequency dependence of $I_{c}$ varies; therefore, further investigations are needed.

The plots between experimental (sphere) and fitted (dashed) lines also the surface leakage noise (line) are shown in Figure 9 for unpassivated and $\mathrm{SiO}_{2}, \mathrm{Si}_{3} \mathrm{~N}_{4}$ passivated SL diodes, respectively.
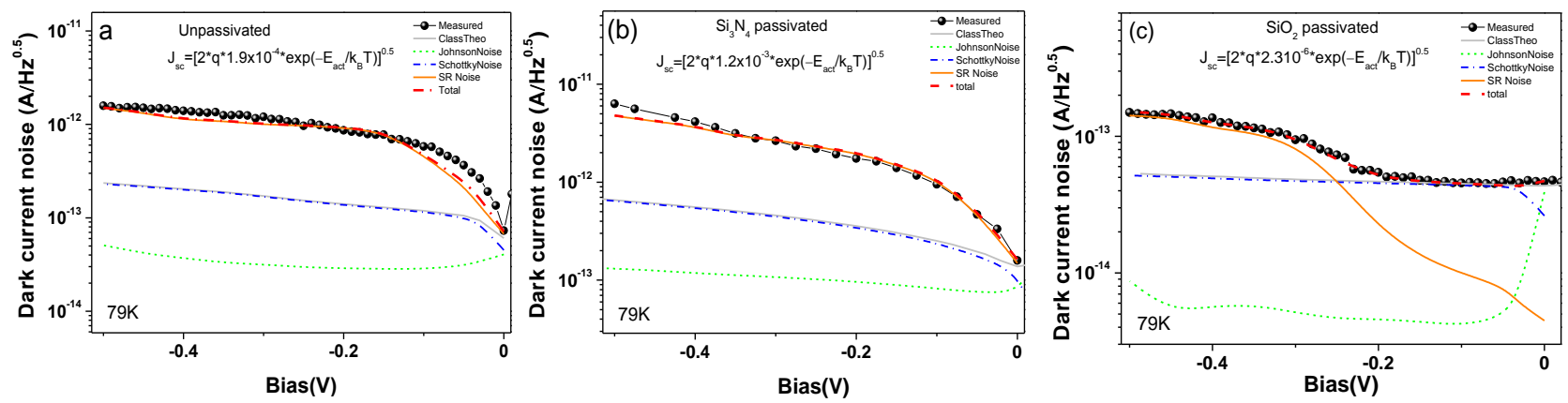

Figure 9. Total dark noise current as function of the bias for the $\mathrm{SL}$ device (a) unpassivated, passivated with (b) $\mathrm{Si}_{4} \mathrm{~N}_{3}$, (c) $\mathrm{SiO}_{2}$, The dashed line shows the calculated Shot-noise; the dotted line represents the Johnson-noise; dashed dotted line demonstrations the calculated surface noise, the lines exhibits the $I_{\text {total }}$ fitted and the white noise

The noise fit is in a good agreement to the experimental results. This agreement between the experimental data and the fit proves that the additional dark noise current is surface recombination current. Thus, it can be concluded that an increase in reverse $\mathrm{V}_{\text {bias }}$ lowers the activation energy barrier at the interfaces exponentially hence generate higher states that contributes to a $1 / \mathrm{f}-$ noise as well as additional to the noise appeared. 


\section{SUMMARY AND CONCLUSION}

In conclusion, we studied the dark current and noise characteristics in MWIR InAs/GaSb SL photodiodes with and without surface passivation. It was shown that $\mathrm{SiO}_{2}$ passivation decreases the dark current by up to two orders of magnitude, which is attributed to the effective suppression of surface leakage currents. The noise measurements revealed the absence of intrinsic 1/f-noise (as well as a noise reduction with one order of magnitude) above $40-30 \mathrm{~Hz}$ and Shottky-limited behavior up to $\mathrm{V}_{\text {Bias }}=\sim-0.15 \mathrm{~V}$. Despite the classical theoretical noise expectation below $\mathrm{V}_{\text {Bias }}=$ $-0.15 \mathrm{~V}$ a difference was observed between the experimental and theoretically calculated Shottky-noise, with a divergence factor of $\sim 3$. The origin of the difference is attributed the presence of surface leakage current, which emanated from the reduction in surface barrier height at the SL interface by the applied reverse $\mathrm{V}_{\text {bias }}$. The leakage current was shown to result from a surface recombination mechanism, due to the relationship between 1/f-noise and the RA vs. 1/T deviations (at a given reverse Bias). This helped to explain the increase in 1/f-noise with reverse $\mathrm{V}_{\text {bias }}$. Indeed, the appearance of additional frequency noise associated with the surface states is consistent with the

observation of surface leakage current that is also attributed to an electrical activity of surface states.

\section{REFERENCES}

[1] A. Rogalski, Science ProgrRess in Quantum Electronics, 27, 59-210 (2003).

[2] R. Chaghi, C. Cervera, H. Ait-Kaci, P. GrRech, J. B. Rodriguez, and P. Christol,

[3] B.K Jones, Adv. Electron. Electron Phys. 87, 201-257 (1993)

[4] Weissman M B,, 1/f-noise"” Rev. Mod. Phys. 60, 537 (1988)

[5] A. Gin, Y. Wei, J. Bae, A. Hood, J. Nah, M. Razeghi, Thin Solid Films, 447, 489-492 (2004).

[6] T. Tansel et. al. IEEE Photon. Technol. Lett., 24, 9, (2012)

[7] K. Jaworowicz, I. Ribet-Mohammed, C. Cervera, J.B. Rodriguez, and P. Christol, IEEE Photonics Technology Letters, 23, 4, (2011)

[8] E. Pils, M.N. Kutty, S. Myers, H.S. Kim, N. Gautam, L.R. Dawson, S. Krishna, Infrared Physics \&Technology 54, 252-257 (2011).

[9] Antoni Rogalski, Krzystof,, Narrow-gap semiconductor photodiodes SPIE Press, Bellingham, Washington.

[10] B.M. Nguyen et. al. Appl. Phys. Lett. 94, 223506 (2009)

[11]E.K. Huang, D. Hoffman, B. Nguyen, P. Delaunay, M. Razeghi, Appl. Phys. Lett., 94, 053506, (2009).

[12] A. Soibel, D. Z.-Y. Ting, C. J. Hill, M. Lee, J. Nguyen, S. A. Keo, J. M. Mumolo, S. D. Gunapala, Appl. Phys. Lett. 96,111102 (2010)

[13] H. S. Kim, E. Plis, A. Khoshakhlagh, S. Myers, N. Gautam, Y. D. Sharma, L. R. Dawson, S. Krishna, S. J. Lee, and S. K. Noh, Appl. Phys. Lett. 96, 033502 (2010).

[14] G. Marre, B. Vinter and V. Berger, Semicond. Scie. Technol. 18, 284-291 (2003)

[15] Shin Mou, Jian V. Li, and Shun Lien Chung J. Appl. Phys. 102. 066103 (2007)

[16] M. Weinelt, m. kutschera,r. Schmidt,c. orth, t. fauster,m. rohlfing Appl. Phys. A 80, 995-1003 (2005)

[17] Charles A. Vergers,, Handbook of Electrical Noise: measurement and Technology" hand book, 2 Sub edition (1987).

[18]E. Bendada. K. Rais, P. Mialhe and J. P. Charles Active and passive Elec. Comp., 21, 61-71, (1998).

[19] John David Vincent, Fundamentals of Infrared Detector Operation and Testing”, Wiley Series in Pure and Applied Optics, (1990)

[20] Tunay Tansel, Kutlu Kutluer, Abdullah Muti, Omer Salihoglu, Atila Aydinli, and Rasit Turan Applied Physics Express 6, 032202 (2013)

O. Salihoglu, A. Muti, K. Kutluer, T. Tansel, R. Turan, Y. Ergun, and A. Aydinli: Appl. Phys. Lett. 101 (2012) 073505 .

O. Salihoglu, A. Muti, K. Kutluer, T. Tansel, R. Turan, and A. Aydinli:J. Phys. D 45 (2012) 365102. 